\title{
Widespread But Regionally Specific Effects of Experimenter- Versus Self-Administered Morphine on Dendritic Spines in the Nucleus Accumbens, Hippocampus, and Neocortex of Adult Rats
}

\author{
TERRY E. ROBINSON, ${ }^{1, *}$ GRAZYNA GORNY, ${ }^{2}$ VIRGINIA R. SAVAGE, ${ }^{1}$ AND BRYAN KOLB ${ }^{2}$ \\ ${ }^{1}$ Department of Psychology and Neuroscience Program, The University of Michigan, Ann Arbor, MI, USA 48109 \\ ${ }^{2}$ Canadian Centre for Behavioural Neuroscience, University of Lethbridge, Lethbridge, AB, Canada T1K 3M4
}

KEY WORDS Golgi; neuron structure; opiates; orbital frontal cortex; medial frontal cortex; parietal cortex; occipital cortex; dendrite; drug abuse

\begin{abstract}
We studied the effects of self-administered (SA) vs. experimenter-administered (EA) morphine on dendritic spines in the hippocampal formation (CA1 and dentate), nucleus accumbens shell (NAcc-s), sensory cortex (Par1 and Oc1), medial frontal cortex (Cg3), and orbital frontal cortex (AID) of rats. Animals in the SA group self-administered morphine in 2-h sessions $(0.5 \mathrm{mg} / \mathrm{kg} / \mathrm{infusion}$, i.v.) for an average of 22 sessions and animals in the EA group were given daily i.v. injections of doses that approximated the total session dose for matched rats in Group SA (average cumulative dose $/$ session of $7.7 \mathrm{mg} / \mathrm{kg}$ ). Control rats were given daily i.v. infusions of saline. One month after the last treatment the brains were processed for Golgi-Cox staining. In most brain regions ( $\mathrm{Cg} 3$, Oc1, NAcc-s) morphine decreased the density of dendritic spines, regardless of mode of administration (although to a significantly greater extent in Group SA). However, only SA morphine decreased spine density in the hippocampal formation and only EA morphine decreased spine density in Par1. Interestingly, in the orbital frontal cortex morphine significantly increased spine density in both Groups SA and EA, although to a much greater extent in Group SA. We conclude: 1) Morphine has persistent (at least 1 month) effects on the density of dendritic spines in many brain regions, and on many different types of cells (medium spiny neurons, pyramidal cells, and granule cells); 2) The effect of morphine on spine density (and presumably synaptic organization) varies as a function of both brain region and mode of drug administration; and 3) The ability of morphine to remodel synaptic inputs in a regionally specific manner may account for the many different long-term sequelae associated with opioid use. Synapse 46:271-279, 2002. ๑ 2002 Wiley-Liss, Inc.
\end{abstract}

\section{INTRODUCTION}

Manipulation of opioid systems in the brain is known to produce morphological changes in the structure of neurons and their dendritic processes. For example, if administered early in development opioid drugs, such as morphine, decrease the complexity of dendrites on cells in the neocortex and hippocampus (Ricalde and Hammer, 1990). The blockade of endogenous opioid receptors has the opposite effect (Hauser et al., 1987, 1989), which may reflect alterations in the influence of endogenous opioids on neuronal development. But opioids also affect neuronal morphology when administered in adulthood. For example, the chronic administration of morphine via a subcutaneous implant decreases the size of dopamine cell bodies in the ventral tegmental area (Sklair-Tavron et al., 1996). In addition, repeated, intermittent i.p. injections of morphine decrease dendritic aborization and the density of dendritic spines on neurons in the nucleus accumbens

Contract grant sponsor: the National Institute on Drug Abuse; Contract grant number: R01 DA13389; Contract grant sponsor: the National Science and Engineering Research Council of Canada.

*Correspondence to: Dr. Terry E. Robinson, Biopsychology Program, Department of Psychology, The University of Michigan, 525 E. University (East Hall), Ann Arbor, MI 48109, USA

E-mail: ter@umich.edu

Received 1 July 2002; Accepted 7 August 2002

DOI 10.1002/syn.10146 
shell (NAcc-s), medial prefrontal cortex, and parietal cortex of rats (Robinson and Kolb, 1999b). These effects of morphine on dendritic structure may reflect a drug-induced reorganization of brain circuits that mediate the actions of morphine and thus may be related to some of the persistent sequelae associated with morphine use.

In all previous preclinical studies on the effects of opioid administration on neuronal structure morphine was administered by an experimenter. In considering the extent to which these studies may inform about the effects of drug use and abuse in humans, however, it is important to recognize that the neurobiological effects of drugs administered by an experimenter are not always the same as those observed when drugs are selfadministered (Dworkin, 1995; Mark et al., 1999; Smith and Dworkin, 1990; Stefanski et al., 1999; Wilson et al., 1994). The purpose of the present experiment, therefore, was to determine if self-administered morphine in rats has similar effects on the density of dendritic spines as experimenter-administered morphine. We compared the effects of self-administered morphine and experimenter-administered morphine on spine density in four brain regions shown previously to be sensitive to morphine: the NAcc-s, prefrontal cortex (medial frontal cortex and orbital frontal cortex), sensory cortex (primary somatosensory and primary visual cortex), and hippocampal formation (CA1 and dentate gyrus). To be sure any changes were relatively persistent, animals were studied 1 month after their last drug treatment. We report that morphine has widespread effects on the density of dendritic spines, but its effects vary greatly both as a function of brain region and as a function of mode of drug administration.

\section{MATERIALS AND METHODS Subjects}

Male Sprague-Dawley rats weighing between 200$225 \mathrm{~g}$ at the beginning of the experiment were housed singly in clear plastic cages in a temperature and humidity controlled room, with a 14:10-h light:dark cycle (lights on at 7:00 AM). After a week all rats were mildly food-deprived to about $90 \%$ of normal body weight by restricting the number of food pellets available in the home cages. Controlled feeding was maintained throughout the self-administration phase of the experiment. Animals were then randomly assigned to one of three groups: a group to be trained to self-administer morphine (SA), one to receive experimenter-administered morphine (EA), and one to receive EA saline (control, Ctl).

\section{Procedures}

Animals in the SA group were first trained to bar press (FR1) for $45 \mathrm{mg}$ Noyes food pellets in an operant chamber (Med Associates). Each chamber had two levers and responses on both levers were recorded but only one had programmed consequences. Each press on the active lever resulted in presentation of a food pellet and a 1.5-sec period during which a cue light over the active lever went off and white noise and the house light were turned on. Each animal was trained daily in one 60-min test session until they acquired stable responding, which took an average of 5 days. During this time animals in the EA and Ctl groups were removed from their home cage each day and placed in a test chamber for $60 \mathrm{~min}$, after which time they were placed back into their home cage.

After animals in the SA group acquired stable responding for food, all animals in all groups underwent surgery to implant a chronic indwelling catheter in the right external jugular vein. The surgical procedures were as described previously (Crombag et al., 1996; Weeks, 1972). Briefly, rats were anesthetized using sodium pentobarbital and the right jugular vein was isolated. A silicone catheter was attached via PE tubing to a backport anchored between the animals' shoulder blades, similar in design to that used by Caine et al. (1993). The other end of the catheter was placed into the vein and tied with silk thread before the incisions were sutured. For the duration of the experiment the catheters were flushed daily with $100 \mu$ l of heparinized saline (30 USP/ml heparin in $0.9 \%$ buffered saline). One day after catheter surgery the catheters were tested for patency by injecting $200 \mu \mathrm{l}$ of thiopental sodium $(20 \mathrm{mg} / \mathrm{ml})$. If the animal lost its righting reflex within $5 \mathrm{sec}$ of infusion the catheter was considered patent. Animals were then allowed to recover from surgery for at least 4 days before behavioral testing.

Four days after surgery animals in the SA group were given a couple of additional days of food training before they were transferred to the drug self-administration protocol. The latter consisted of daily 2 -h sessions, Monday through Friday, using an FR1 schedule. During each session the animals were placed into the operant chambers and connected by their backport to tubing attached to a motorized syringe. Each press on the active lever resulted in an i.v. infusion $0.5 \mathrm{mg} / \mathrm{kg}$ of morphine sulfate (weight of the salt) in $50 \mu$ l of phosphate-buffered saline (PBS) over 2.8 sec. During the subsequent 20 -sec time out period, which began immediately upon initiation of a morphine infusion, the house light and white noise were turned on, the cue light over the lever was turned off, and additional lever presses had no programmed consequences during this time. Once animals acquired morphine self-administration behavior, which took 1-3 days, the schedule was increased to FR2 for the remainder of the experiment. Testing continued for a minimum of 13 sessions and a maximum of 30 sessions (see below).

One day after animals in the SA group started selfadministration training each animal in the EA group was paired with a specific animal in the SA group. During the self-administration sessions animals in the EA and Ctl groups were removed from their home cage and transported to a test chamber, where they received 
an i.v. infusion of either morphine (EA) or PBS (Ctl). The dose for each animal in the EA group consisted of the total dose self-administered by its matched SA animal the previous day. On a given day, if this dose was below $2-3 \mathrm{mg} / \mathrm{kg}$ it was given as a single bolus infusion in $50 \mu \mathrm{l}$ of PBS. When the dose to be administered was larger, however, there was a danger of a lethal overdose. In these cases $10 \mathrm{mg}$ of morphine per $200 \mu \mathrm{l}$ of PBS was given in multiple bolus infusions over a greater period of time (12-18 min using a motorized pump) and the number of injections depended on the total dose required. For example, if a total dose of $9 \mathrm{mg} / \mathrm{kg}$ was required it was given in three separate injections of $3 \mathrm{mg} / \mathrm{kg}$ each spaced over a total of 10-12 min. This procedure ensured that the total dose received each day by animals in the EA group was the same as animals in the SA group, although, of course, this did not match the pattern of administration or pharmacokinetic profile. This procedure was used because we were primarily interested in comparing animals given injections by an experimenter, in a similar fashion as might occur in a typical sensitization experiment, with animals that self-administered morphine, but at the same time controlling for at least the total dose received each day. Animals in the Ctl group were paired with one of the animals in the EA group and received an equal volume of PBS. After their injections animals in the EA and Ctl groups were left in the test cages for $2 \mathrm{~h}$ before they were returned to their home cage.

Four rats in the SA group maintained patent catheters for at least 2 weeks of self-administration testing and data analysis is based on these four animals in the SA group, on four matched animals in the EA group and four animals in the Ctl group. Of the four rats in the SA group one was tested for 13 sessions, one for 17 sessions, one for 28 sessions, and one for 30 sessions. The mean $( \pm$ SEM) intake of morphine/session for these animals was $7.66 \pm 1.2 \mathrm{mg} / \mathrm{kg}$ (range, $5.15-10.8 \mathrm{mg} /$ $\mathrm{kg} / \mathrm{session}$ ). The mean dose/session for rats in the EA group was $7.73 \pm 0.59 \mathrm{mg} / \mathrm{kg}$. All rats received close to their average dose/session with 2-3 days of training. In most cases where testing was discontinued it was because the catheter ceased to be patent.

\section{Anatomical procedures}

Following the final test session, all animals were left undisturbed for 1 month. After this the animals were given a lethal injection of sodium pentobarbital and then were perfused through the heart with $0.9 \%$ saline. The brains were removed and placed in light-tight vials containing Golgi-Cox solution (Gibb and Kolb, 1998). After 14 days the brains were transferred to a $30 \%$ sucrose solution for at least 3 days, before coronal sections $(200 \mu \mathrm{m})$ were obtained using a vibrating microtome. The sections were placed on $2 \%$ gelatin-coated slides and stained using procedures described in detail by Gibb and Kolb (1998).
Cells in four brain regions were selected for analysis. These regions were chosen because in previous studies all have been shown to undergo plastic changes either in response to repeated exposure to drugs of abuse or to other kinds of experiences. The regions/cells that were examined included (terminology according to Zilles, 1985; Zilles et al., 1990): 1) hippocampal formation (pyramidal cells in the CA1 and dentate granule cells). 2) Sensory cortex, including pyramidal cells in layer III of primary somatosensory cortex (Par1) and pyramidal cells in layer III of primary visual cortex (Oc1). 3) Prefrontal cortex, including pyramidal cells in layer V of the medial frontal cortex (Cg3) and pyramidal cells in layer III of the orbital region (specifically, area AID, agranular insular cortex). These are the two portions of rat frontal cortex that receive inputs from the mediodorsal nucleus of the thalamus (which defines them as prefrontal cortex), and they are also the two main dopamine terminal regions in rat frontal cortex (Kolb, 1990; Lindvall et al., 1978). 4) Nucleus accumbens (NAcc-s, medium spiny neurons in the shell).

For each region/cell type we calculated the density of dendritic spines on terminal dendrites and for pyramidal cells separate measurements were obtained for apical and basilar dendrites. The relevant brain region was first identified at low power $(100 \times)$ and then specific cells from each hemisphere identified at $250 \times$. In order to be included in the analysis the dendritic tree of a cell had to be intact (i.e., largely in the $200 \mu \mathrm{m}$ section and not obscured by blood vessels or astrocytes). Spine density was calculated by using camera lucida to trace a length of dendrite (at least $20 \mu \mathrm{m}$ long) at 1,000×. The exact length of the dendritic segment was calculated and the number of spines along the entire length counted. For apical dendrites of Par1, Oc1, AID, Cg3, and CA1 pyramidal cells spines were counted on one third-order terminal tip (i.e., a terminal branch). For basilar dendrites of Par1, Oc1, AID, and Cg3 pyramidal cells spines were counted on one fourth-order terminal tip (and from a third-order terminal tip for CA1 cells). For medium spiny neurons, spines were counted on a third-order terminal tip. For dentate granule cells, spines were counted on second- or third-order terminal dendrites. One dendrite was examined per neuron. No attempt was made to correct for the fact that spines directly below and above the plane of view could not be seen, so this measure necessarily underestimates total spine number. The values for at least five cells in each hemisphere of each rat were averaged and hemisphere was used as the unit of analysis. All quantification was performed by the same person (G.G.), who was blind to experimental condition.

\section{RESULTS}

In the NAcc-s, Oc1 and Cg3 morphine significantly decreased the density of dendritic spines regardless of mode of drug administration, although in some regions (NAcc-s, Oc1-apical, Cg3-basilar) the effect of self-ad- 


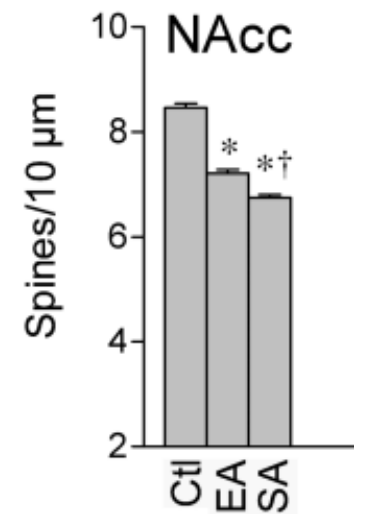

Fig. 1. The density of dendritic spines (spines/10 $\mu \mathrm{m}$ ) on medium spiny neurons in the shell of the nucleus accumbens of rats given intravenous saline (Ctl), experimenter-administered morphine (EA), or self-administered morphine (SA). The data were analyzed using analysis of variance and Scheffé tests were used for pairwise comparisons. There were significant group differences $(\mathrm{F}[2,21]=136, P<$ 0.001). *Significantly different from the Ctl group; 'Significantly different from the EA group (Scheffé tests).

\section{Prefrontal Cortex}

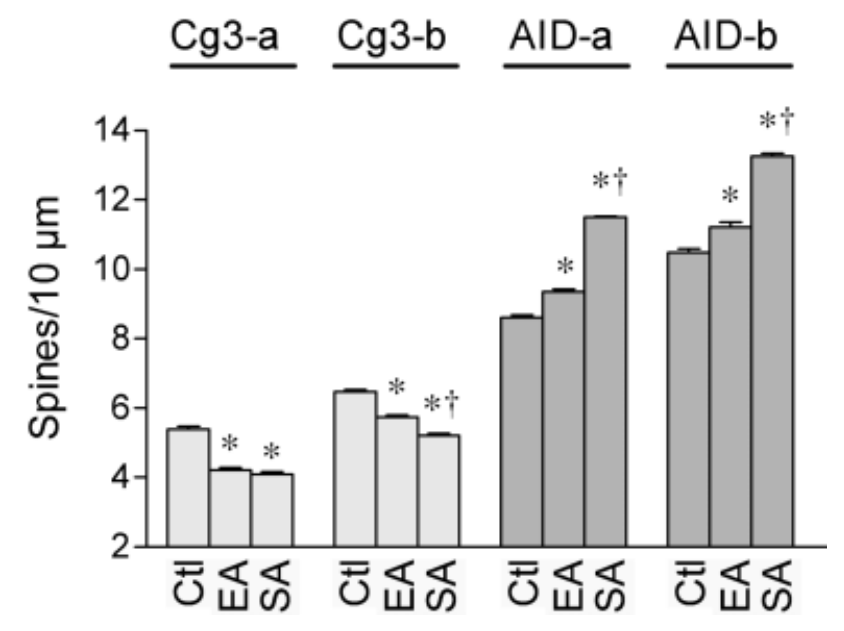

Fig. 2. The density of dendritic spines (spines/10 $\mu \mathrm{m}$ ) in two prefrontal cortical regions, layer V pyramidal cells in Cg3 and layer III pyramidal cells in AID (terminology of Zilles, 1985). These are the two major frontal areas to receive input from the mediodorsal thalamus and dopamine projections from the midbrain. Rats were given intravenous saline (Ctl), experimenter-administered morphine (EA), or self-administered morphine (SA). The "a" suffix refers to apical dendrites and the "b" suffix to basilar dendrites. There were significant group differences: $\mathrm{Cg} 3-\mathrm{a}, \mathrm{F}=85.8, P<0.001 ; \mathrm{Cg} 3-\mathrm{b}, \mathrm{F}=116, P<$ 0.001 ; AID-a, $\mathrm{F}=265, P<0.001$; AID-b, $\mathrm{F}=135, P<0.001$. *Significantly different from the Ctl group; ${ }^{\dagger}$ Significantly different from the EA group (Scheffé tests).

ministered morphine was significantly greater than the effect of experimenter-administered morphine (Figs. 1, 2, 3; see figure legends for statistics). In Par1, however, morphine decreased spine density only in animals that were administered morphine by the experimenter; there was no effect of self-administered morphine on spine density on either the apical or basilar dendrites of pyramidal cells in Par1 (Fig. 3). In contrast, the opposite pattern was seen in the hip-

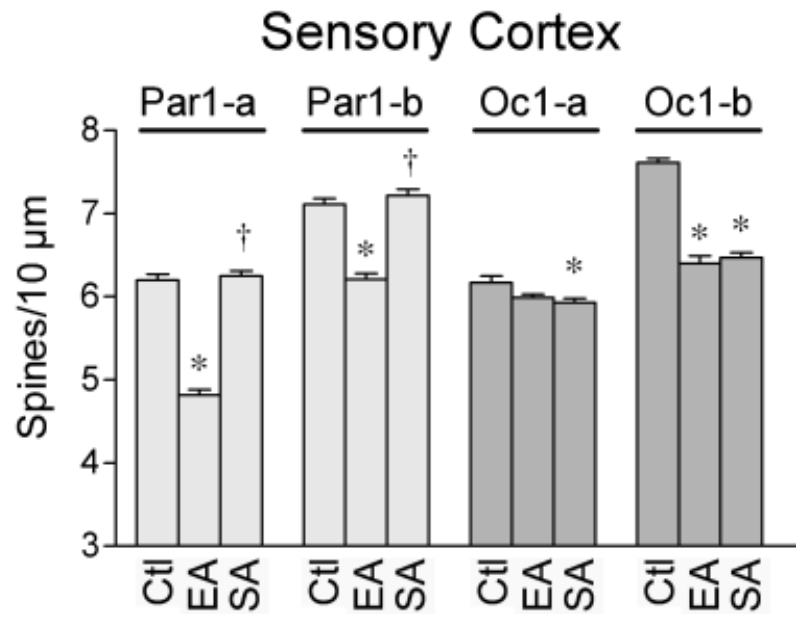

Fig. 3. The density of dendritic spines (spines/10 $\mu \mathrm{m}$ ) on layer III pyramidal cells in primary somatosensory cortex (Par1) and primary visual cortex (Oc1). The "a" suffix refers to apical dendrites and the "b" suffix to basilar dendrites. There were significant group differences: Par1-a, $\mathrm{F}=173, P<0.001 ;$ Par1-b, $\mathrm{F}=63.7, P<0.001$; Oc1-a, $\mathrm{F}=5.28, P<0.02 ;$ Oc1-b, $\mathrm{F}=39.4, P<0.001$. *Significantly different from the Ctl group; 'Significantly different from the EA group (Scheffé tests).

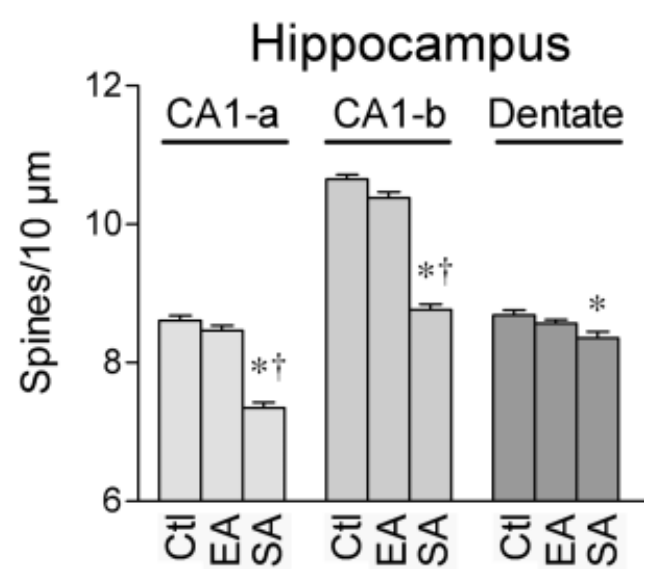

Fig. 4. The density of dendritic spines (spines/10 $\mu \mathrm{m}$ ) on CA1 pyramidal cells and dentate granule cells in the hippocampal formation. The "a" suffix refers to apical dendrites and the "b" suffix to basilar dendrites. There were significant group differences: CA1-a, $\mathrm{F}=90.0, P<0.001$; CA1-b, $\mathrm{F}=187, P<0.001$; dentate, $\mathrm{F}=5.19$, $P<0.02$. *Significantly different from the Ctl group; 'Significantly different from the EA group (Scheffé tests).

pocampal formation. Morphine significantly decreased spine density on CA1 pyramidal cells and dentate granule cells only in animals that self-administered morphine; the effect of experimenter-administered morphine was nonsignificant (Fig. 4).

The effect of morphine on pyramidal cells in AID differed from that seen in any of the other brain regions studied. In AID morphine significantly increased spine density on both apical and basilar dendrites of pyramidal cells, whether morphine was self-administered or administered by the experimenter (although the effect of self-administered morphine was much larger than the effect of experimenter-administered morphine; Fig. 2). 


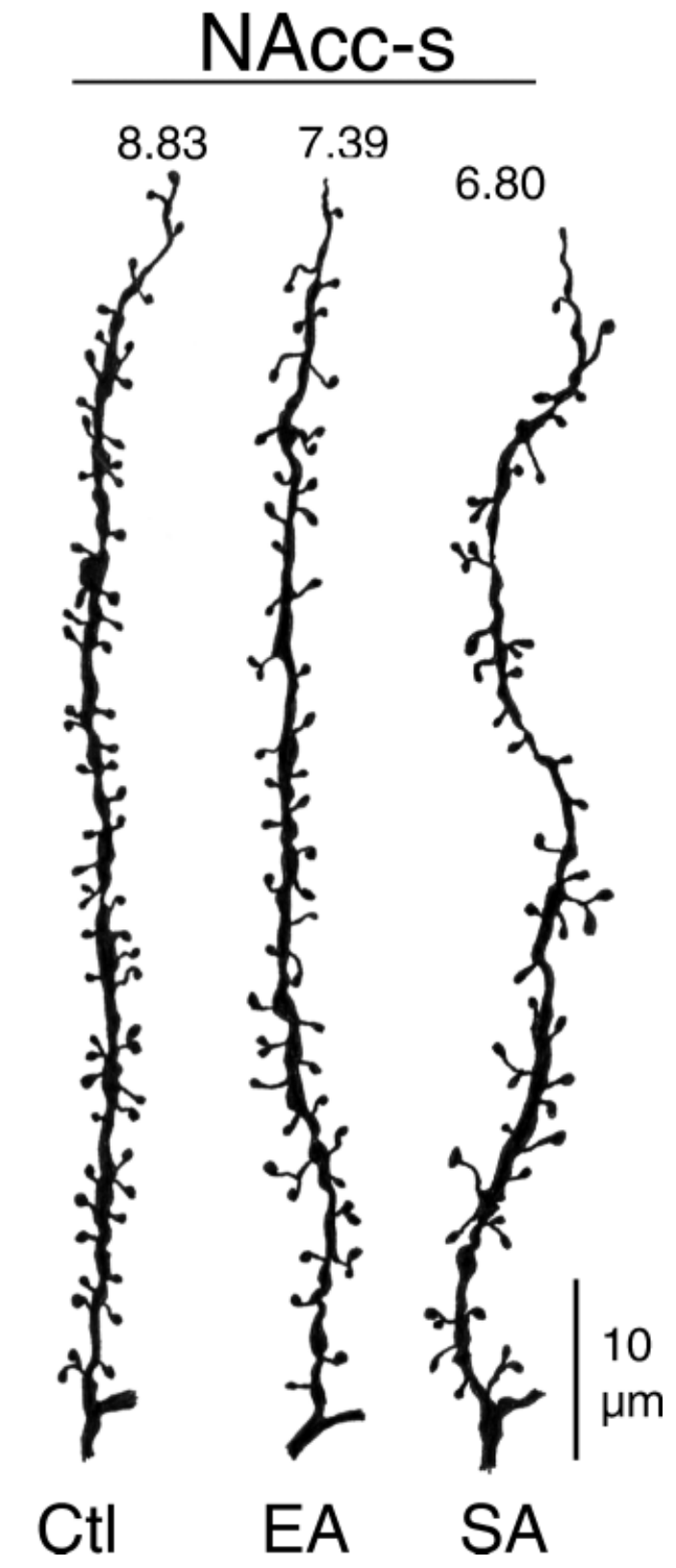

Fig. 5. Representative camera lucida drawings $(2,000 \times)$ of dendrites on medium spiny neurons in the nucleus accumbens shell (NAcc-s) of rats given intravenous saline (Ctl), experimenter-administered morphine (EA), or self-administered morphine (SA). The numbers at the top of each dendritic segment indicate the calculated numbers of spines $10 \mu \mathrm{m}$ for the specific dendrite illustrated. The scale bar indicates a distance of $10 \mu \mathrm{m}$. Note the difference in the shape of spines between the Ctl and the EA and SA groups. Many spines in morphine treated animals (especially the SA group) appear elongated relative to $\mathrm{Ctl}$, despite the reduction in spine density.

In addition to these effects of morphine on the density of dendritic spines, we also observed some qualitative effects. In the NAcc-s and Cg3, where morphine decreased spine density, many spines appeared to be appreciably elongated, that is, have longer stems than in control animals. This effect is apparent in Figures $5-7$, although it was not quantified. In contrast, in AID, where morphine increased spine density, many spines appeared to be relatively stubby, to have shorter stems than normal (see Fig. 7). We did not observe any obvious changes in the shape of spines in the hippocampal formation, Par1 or Oc1 (see Fig. 8).

\section{DISCUSSION}

We reported previously that repeated, intermittent i.p. injections of morphine produce a persistent (25 days after the last morphine treatment) decrease in dendritic branching and spine density on medium spiny neurons in the NAcc-s and pyramidal cells in Par1 and Cg3 (Robinson and Kolb, 1999b). Consistent with this, we report here that repeated experimenteradministered intravenous injections of morphine also decreased spine density in the NAcc-s, Par1, and Cg3 (as well as on the basilar dendrites of Oc1 pyramidal cells). As in our earlier study, this effect was evident long ( 1 month) after the last exposure to morphine. This apparent widespread reduction in dendritic complexity produced by morphine is consistent with reports that, when given early in development, morphine decreases dendritic growth in the cerebral cortex and hippocampus (Ricalde and Hammer, 1990). This latter effect may be because morphine interferes with the action of endogenous opioids on neuronal development, because the blockade of opioid receptors in developing animals has the opposite effect as morphine (Hauser et al., 1987, 1989). Taken together, these data suggest that endogenous opioid systems play an important role in maintaining and regulating the structure of dendrites and dendritic spines, both in development and in adulthood. They further suggest that some of the longterm effects of opioid drugs may be due to their ability to remodel dendrites, which presumably reflects a drug-induced reorganization of synaptic inputs onto these dendrites.

It is possible that the morphine-induced changes in dendritic spines described here are related to reports that repeated exposure to morphine in adulthood can compromise neuronal integrity in widespread regions of the brain. For example, repeated treatment with morphine alters neurofilament and other cytoskeletal proteins in the cerebral cortex and ventral tegmental area (VTA), both in animals (Beitner-Johnson et al., 1992a; Boronat et al., 1998; Jaquet et al., 2001) and human opioid addicts (Ferrer-Alcon et al., 2000; Garcia-Sevilla et al., 1997), decreases axonal transport in the VTA-NAcc system (Beitner-Johnson and Nestler, 1993) and decreases the size of dopamine cell bodies in the VTA (Sklair-Tavron et al., 1996). Chronic morphine also increases the expression of the pro-apoptotic Fas receptor and decreases the expression of the anti-apoptotic Bcl-2 oncoprotein in the cerebral cortex of rats (Boronat et al., 2001), which may be related to a morphine-induced inhibition of neurogenesis in the dentate granule cell layer (Eisch et al., 2000) and/or the decrease in frontal lobe volume seen in opioid addicts (Pezawas et al., 1998). The overall picture emerging 


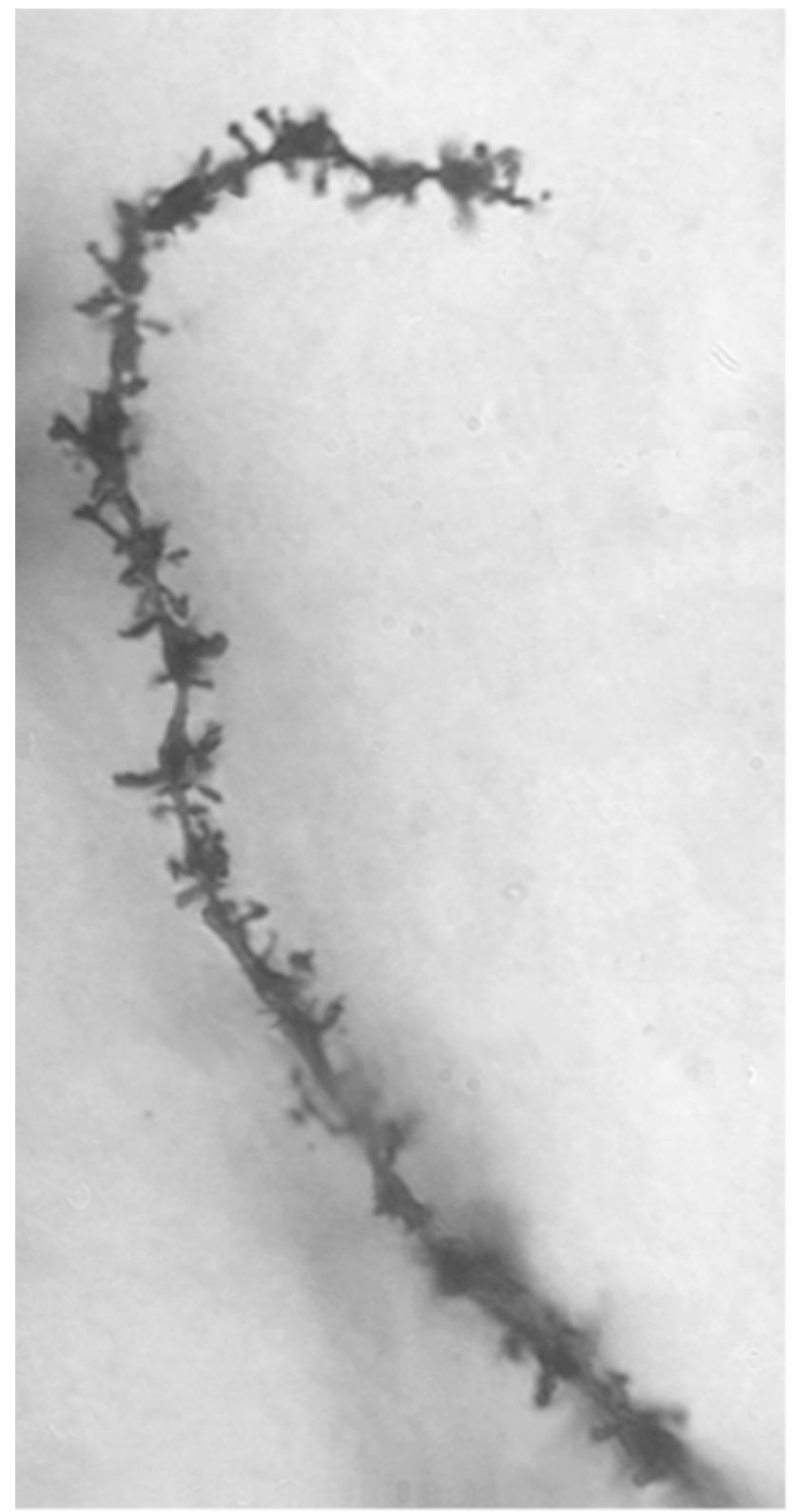

Ctl

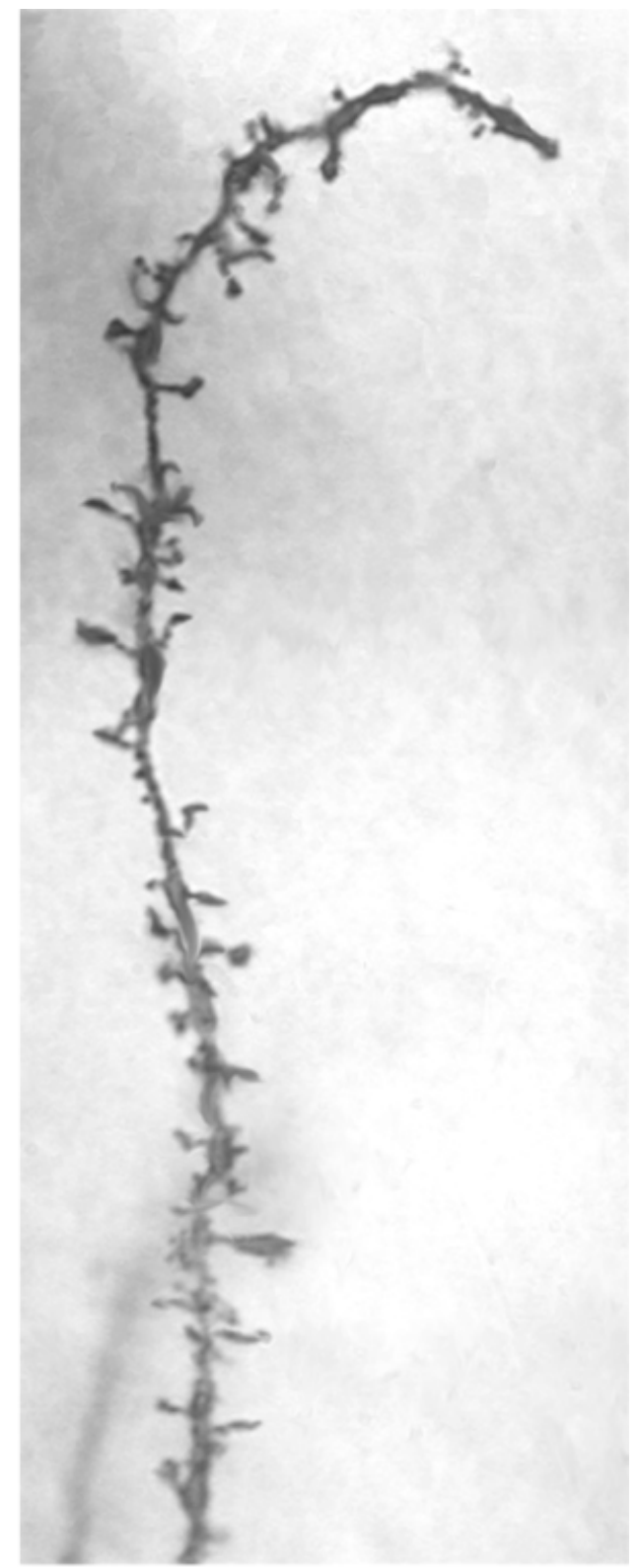

SA

Fig. 6. Photomicrographs of representative dendrites from NAcc-s medium spiny neurons illustrating spines in a control animal (Ctl) and an animal that self-administered morphine (SA).

from these studies is that repeated exposure to morphine damages neurons (Nestler, 1996).

On the other hand, the data presented here suggest that morphine does not have generalized neurodegenerative effects (at least as indicated by changes in spine density), because its effects on dendritic structure were not uniform across different brain regions, nor mode of drug administration. In some brain regions (NAcc-s, Cg3, Oc1) morphine did decrease spine density, regardless of mode of drug administration, and the effect of self-administered morphine was generally greater than the effect of experimenter-administered morphine. But in the hippocampal formation (CA1 and dentate gyrus) morphine decreased spine density only when it was self-administered, and in Par1 morphine decreased spine density only when it was experimenter-administered. The different effects of morphine in different subregions of the prefrontal cortex were especially striking. As noted above, in the medial frontal cortex (Cg3) morphine decreased spine density, but in a closely related orbital frontal region (AID) morphine produced a large increase in spine density, which was especially dramatic in animals with self-administration experience. 

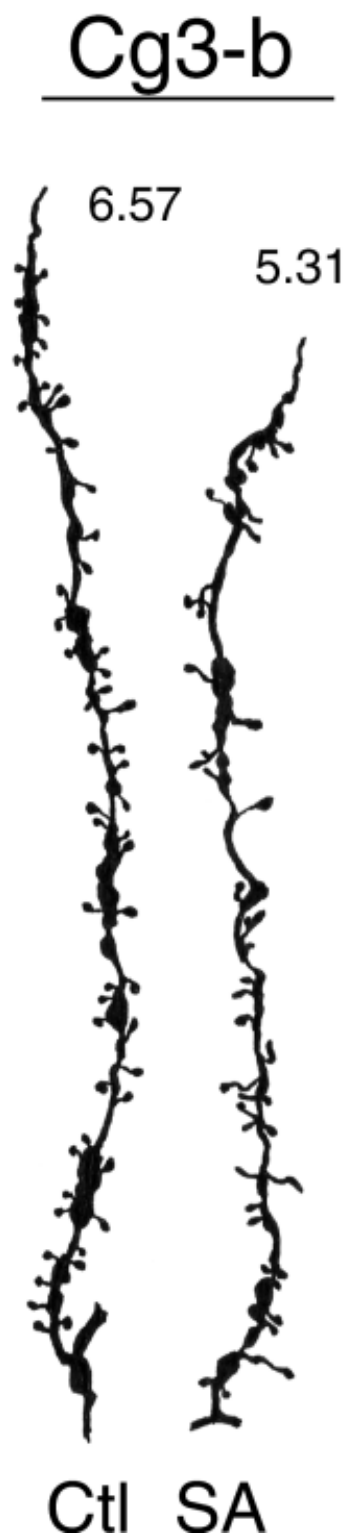

AID-a

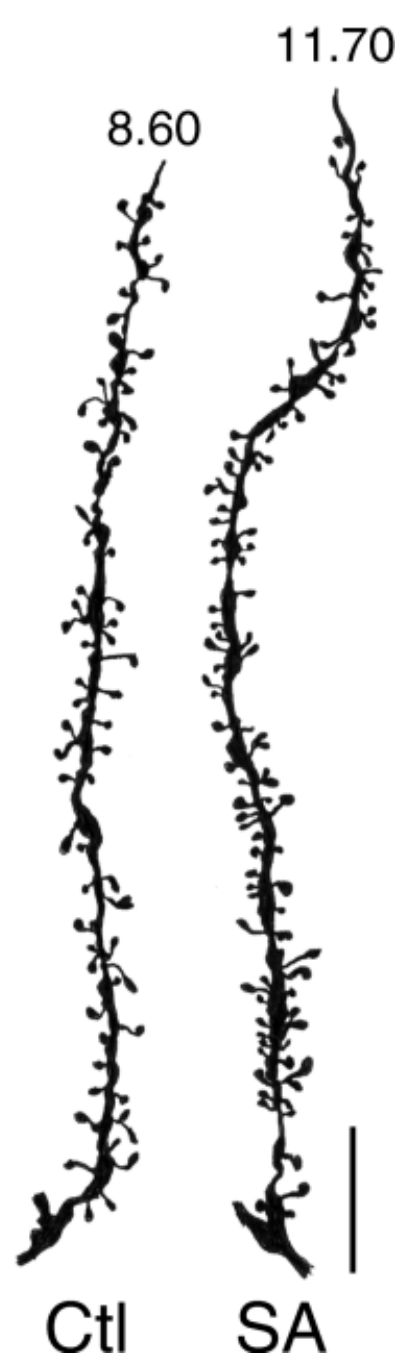

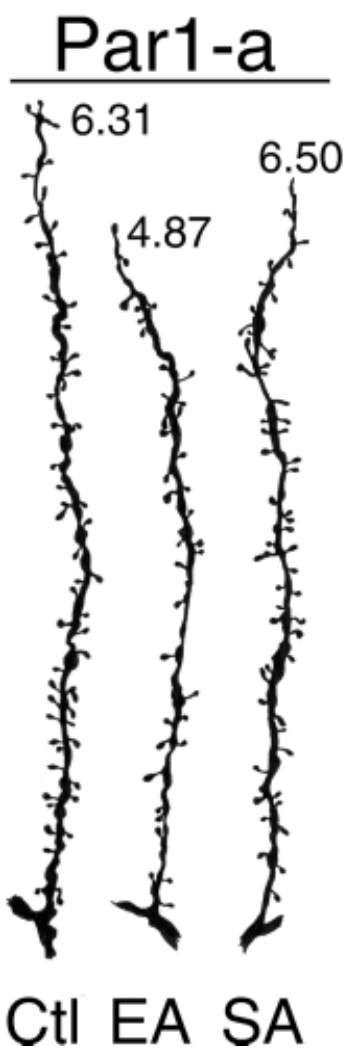

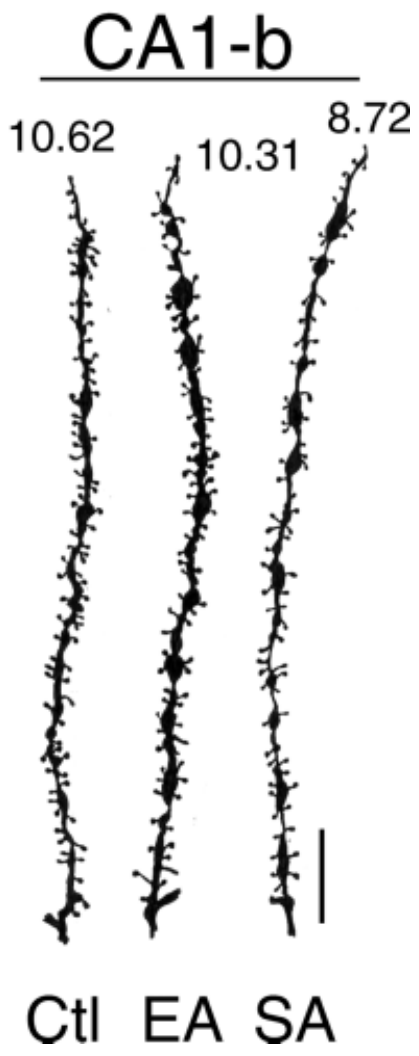

Fig. 8. Representative camera lucida drawings $(2,000 \times)$ of dendrites from pyramidal cells in Par1-a (where there was a decrease in spine density only in the EA group), and CA1-b pyramidal cells in the hippocampal formation (where there was a decrease in spine density only in the SA group). Note that there are no obvious group differences in the shape of the spines. Scale bar $=10 \mu \mathrm{m}$.

which would maintain relatively high blood levels of the drug for a much more sustained period of time. It is possible that sustained blood levels might be more toxic, as is the case with psychostimulant drugs (Robinson and Becker, 1986). But self-administered morphine did not consistently produce a larger effect than experimenter-administered drug. In Par1 only experimenter-administered morphine had any effect, and in some regions there was no difference in the effect of experimenter- and self-administered morphine (e.g., Cg3-a, Oc1-b). The fact that different modes of drug administration has such different effects in some brain regions is consistent with previous reports that selfadministered drugs and experimenter-administered drugs sometimes have different neurobiological effects (Dworkin, 1995; Mark et al., 1999; Smith and Dworkin, 1990; Stefanski et al., 1999; Wilson et al., 1994).

The regional specificity of the effects described here has important implications in thinking about the mechanisms by which morphine, and associated changes in opioid neurotransmission, shape the structure of dendrites. The results suggest that repeated treatment with morphine does not alter spine density due to the action of morphine on some ubiquitous, brain-wide growth factor. To the extent that the effect 
of morphine on spine density is mediated by its effect on growth factors (Messer et al., 2000), neurofilament proteins (Beitner-Johnson et al., 1992b; Boronat et al., 1998), or other molecules that regulate neuronal and synaptic structure (Nestler, 1996), the actions of such agents must also vary regionally, and as a function of mode of drug administration. Indeed, the specificity of the effects described here may provide an excellent avenue to delineate mechanisms that are causally related to the action of morphine on dendritic structure. If one hypothesizes, for example, that a given molecule is critical for morphine to alter synaptic organization (at least as indicated by changes in spine density), then in the Par1 only experimenter-administered morphine should have an effect on the molecule under study, in the hippocampal formation only self-administered morphine should have an effect, and in Cg3 and AID one might predict opposite effects. Of course, it is also possible that the effects of morphine on synaptic organization in different types of cells in different brain regions are mediated by entirely different mechanisms. Whatever the case, it is clear that in studies exploring the molecular mechanisms responsible for shaping the structure of dendrites, and associated patterns of synaptic connectivity, it will be important to study very specific brain regions and cell types, and perhaps even specific portions of a dendritic tree, and to not lump samples into broad anatomical categories, such as "the cerebral cortex."

The alterations in spine density reported here presumably reflect a reorganization of synapses on these cells (Harris and Kater, 1994; Shephard, 1996), although this cannot be established with certainty without additional ultrastructural studies. Nevertheless, there is considerable evidence from studies of other forms of experience-dependent plasticity that the kinds of changes seen here in Golgi-stained material are associated with alterations in patterns of synaptic connectivity (Greenough et al., 1990; Ingham et al., 1998; Kolb et al., 1998; Purves, 1994; Woolley, 1999). Given that the changes in dendritic spines do reflect a reorganization of synaptic inputs onto these cells, they must have functional consequences for the operation of the neural systems in which these cells are embedded. We can only speculate what the functional consequences might be. Repeated exposure to morphine has many persistent effects on behavior. For example, repeated treatment with morphine produces a persistent increase (sensitization) in the behavioral activating and the incentive properties of the drug (De Vries and Shippenberg, 2002; Robinson and Berridge, 2000), which could be related to the reorganization of NAcc and prefrontal circuits. There is also accumulating evidence for "distinct patterns of cognitive impairment" in opioid addicts (Ornstein et al., 2000; Rogers et al., 1999; Rogers and Robbins, 2001), which could be related to remodeling of the hippocampal formation and frontal cortex. Changes in the organization of sensory cortical areas may even contribute to deficits on some learning tasks seen in addicts, such as tactual performance tasks (Hill and Mikhael, 1979), due to changes in sensory functions.

In conclusion, and as put by Ornstein et al. (2000), the challenge for the future will be in "matching such patterns of anatomical connectivity to similar brain changes in human drug users and associated, possibly drug-specific, changes in cognitive function" (p. 124), and, we would add, in incentive motivational function. This challenge is further complicated by the fact that the effects of other drugs of abuse on dendritic structure are very different than described here for morphine (Brown and Kolb, 2001; Robinson and Kolb, 1997, 1999a,b; Robinson et al., 2001). The challenge, therefore, will be in unraveling the relationship between alterations in patterns of synaptic connectivity in a specific circuit to the operation of the circuit, and finally to associated changes in behavioral or psychological functions. Nevertheless, the data presented here suggest that repeated exposure to morphine initiates a widespread reorganization of brain regions that mediate cognitive functions, including those associated with judgment and impulsivity, as well as brain regions associated with incentive motivational functions, and together these may contribute to the development of the compulsive patterns of drug-seeking and drug-taking behavior that characterize addiction (Jentsch and Taylor, 1999; Robinson and Berridge, 2000, 2003; Rogers and Robbins, 2001).

\section{ACKNOWLEDGMENTS}

TER was supported by a Senior Scientist Award (K05 DA00473).

\section{REFERENCES}

Beitner-Johnson D, Nestler EJ. 1993. Chronic morphine impairs axoplasmic transport in the rat mesolimbic dopamine system. Neuroreport 5:57-60.

Beitner-Johnson D, Guitart X, Nestler EJ. 1992a. Common intracellular actions of chronic morphine and cocaine in dopaminergic brain reward regions. Ann NY Acad Sci 654:70-87.

Beitner-Johnson D, Guitart X, Nestler EJ. 1992b. Neurofilament proteins and the mesolimbic dopamine system: common regulation by chronic morphine and chronic cocaine in the rat ventral tegmental area. J Neurosci 12:2165-2176.

Boronat MA, Olmos G, Garcia-Sevilla JA. 1998. Attenuation of tolerance to opioid-induced antinociception and protection against morphine-induced decrease of neurofilament proteins by idazoxan and other I2-imidazoline ligands. Br J Pharmacol 125:175-185.

Boronat MA, Garcia-Fuster MJ, Garcia-Sevilla JA. 2001. Chronic morphine induces up-regulation of the pro-apoptotic Fas receptor and down-regulation of the anti-apoptotic Bcl-2 oncoprotein in rat brain. Br J Pharmacol 134:1263-1270.

Brown RW, Kolb B. 2001. Nicotine sensitization increases dendritic length and spine density in the nucleus accumbens and cingulate cortex. Brain Res 899:94-100.

Caine SB, Lintz R, Koob GF. 1993. Intravenous drug self-administration techniques in animals. In: Sahgal A, editor. Behavioral neuroscience, a practical approach, vol. II. New York: IRL Press at Oxford University Press. p 117-143.

Crombag HS, Badiani A, Robinson TE. 1996. Signalled versus unsignalled intravenous amphetamine: large differences in the acute psychomotor response and sensitization. Brain Res 722:227-231.

De Vries TJ, Shippenberg TS. 2002. Neural systems underlying opiate addiction. J Neurosci 22:3321-3325. 
Dworkin SI, Mirkis S, Smith JE. 1995. Response-dependent versus response-independent presentation of cocaine: differences in the lethal effects of the drug. Psychopharmacology 117:262-266.

Eisch AJ, Barrot M, Schad CA, Self DW, Nestler EJ. 2000. Opiates inhibit neurogenesis in the adult rat hippocampus. Proc Natl Acad Sci USA 97:7579-7584.

Ferrer-Alcon M, Garcia-Sevilla JA, Jaquet PE, La Harpe R, Riederer BM, Walzer C, Guimon J. 2000. Regulation of nonphosphorylated and phosphorylated forms of neurofilament proteins in the prefrontal cortex of human opioid addicts. J Neurosci Res 61:338-349.

Garcia-Sevilla JA, Ventayol P, Busquets X, La Harpe R, Walzer C, Guimon J. 1997. Marked decrease of immunolabelled $68 \mathrm{kDa}$ neurofilament (NF-L) proteins in brains of opiate addicts. Neuroreport 8:1561-1565.

Gibb R, Kolb B. 1998. A method for vibratome sectioning of Golgi-Cox stained whole rat brain. J Neurosci Meth 79:1-4.

Greenough WT, Withers GS, Wallace CS. 1990. Morphological changes in the nervous system arising from behavioral experience: what is the evidence that they are involved in learning and memory? In: Squire LR, Lindenlaub E, editors. The biology of memory, Symposia Medica Hoechst. New York: F. K. Schattauder Verlag. p 159-185.

Harris KM, Kater SB. 1994. Dendritic spines: cellular specializations imparting both stability and flexibility to synaptic function. Annu Rev Neurosci 17:341-371

Hauser KF, McLaughlin PJ, Zagon IS. 1987. Endogenous opioids regulate dendritic growth and spine formation in developing rat brain. Brain Res 416:157-161.

Hauser KF, McLaughlin PJ, Zagon IS. 1989. Endogenous opioid systems and the regulation of dendritic growth and spine formation. J Comp Neurol 281:13-22.

Hill SY, Mikhael MA. 1979. Computerized transaxial tomographic and neuropsychological evaluations in chronic alcoholics and heroin abusers. Am J Psychiatry 136:598-602.

Ingham CA, Hood SH, Taggart P, Arbuthnott GW. 1998. Plasticity of synapses in the rat neostriatum after unilateral lesion of the nigrostriatal dopaminergic pathway. J Neurosci 18:4732-4743.

Jaquet PE, Ferrer-Alcon M, Ventayol P, Guimon J, Garcia-Sevilla JA. 2001. Acute and chronic effects of morphine and naloxone on the phosphorylation of neurofilament-H proteins in the rat brain. Neurosci Lett 304:37-40.

Jentsch JD, Taylor JR. 1999. Impulsivity resulting from frontostriatal dysfunction in drug abuse: implications for the control of behavior by reward-related stimuli. Psychopharmacology 146:373-390.

Kolb B. 1990. Organization of the neocortex of the rat. In: Kolb B, Tees RC, editors. The cerebral cortex of the rat. Cambridge, MA: MIT Press. p 21-33.

Kolb B, Forgie M, Gibb R, Gorny G, Rowntree S. 1998. Age, experience and the changing brain. Neurosci Biobehav Rev 22:143-159.

Lindvall O, Bjorklund A, Divac I. 1978. Organization of catecholamine neurons projecting to the frontal cortex in the rat. Brain Res 142: $1-24$.

Mark GP, Hajnal A, Kinney AE, Keys AS. 1999. Self-administration of cocaine increases the release of acetylcholine to a greater extent than response-independent cocaine in the nucleus accumbens of rats. Psychopharmacology (Berl) 143:47-53.

Messer CJ, Eisch AJ, Carlezon WA Jr, Whisler K, Shen L, Wolf DH, Westphal H, Collins F, Russell DS, Nestler EJ. 2000. Role for GDNF in biochemical and behavioral adaptations to drugs of abuse. Neuron 26:247-257.

Nestler EJ. 1996. Under siege: the brain on opiates. Neuron 16:897900.

Ornstein TJ, Iddon JL, Baldacchino AM, Sahakian BJ, London M, Everitt BJ, Robbins TW. 2000. Profiles of cognitive dysfunction in chronic amphetamine and heroin abusers. Neuropsychopharmacology $23: 113-126$.
Pezawas LM, Fischer G, Diamant K, Schneider C, Schindler SD Thurnher M, Ploechl W, Eder H, Kasper S. 1998. Cerebral CT findings in male opioid-dependent patients: stereological, planimetric and linear measurements. Psychiatry Res 83:139-147.

Purves D. 1994. Neural activity and the growth of the brain. Cambridge, UK: Cambridge University Press.

Ricalde AA, Hammer RP Jr. 1990. Perinatal opiate treatment delays growth of cortical dendrites. Neurosci Lett 115:137-143.

Robinson TE, Becker JB. 1986. Enduring changes in brain and behavior produced by chronic amphetamine administration: a review and evaluation of animal models of amphetamine psychosis. Brain Res Rev 11:157-198.

Robinson TE, Berridge KC. 2000. The psychology and neurobiology of addiction: an incentive-sensitization view. Addiction 95(Suppl. 2): S91-S117.

Robinson TE, Berridge KC. 2003. Addiction. Ann Rev Psychol (in press).

Robinson TE, Kolb B. 1997. Persistent structural modifications in nucleus accumbens and prefrontal cortex neurons produced by previous experience with amphetamine. J Neurosci 17:8491-8497.

Robinson TE, Kolb B. 1999a. Alterations in the morphology of dendrites and dendritic spines in the nucleus accumbens and prefrontal cortex following repeated treatment with amphetamine or cocaine. Eur J Neurosci 11:1598-1604.

Robinson TE, Kolb B. 1999b. Morphine alters the structure of neurons in the nucleus accumbens and neocortex of rats. Synapse 33:160162.

Robinson TE, Gorny G, Mitton E, Kolb B. 2001. Cocaine self-administration alters the morphology of dendrites and dendritic spines in the nucleus accumbens and neocortex. Synapse 39:257-266.

Rogers RD, Robbins TW. 2001. Investigating the neurocognitive deficits associated with chronic drug misuse. Curr Opin Neurobiol $11: 250-257$

Rogers RD, Everitt BJ, Baldacchino A, Blackshaw AJ, Swainson R, Wynne K, Baker NB, Hunter J, Carthy T, Booker E, London M, Deakin JF, Sahakian BJ, Robbins TW. 1999. Dissociable deficits in the decision-making cognition of chronic amphetamine abusers, opiate abusers, patients with focal damage to prefrontal cortex, and tryptophan-depleted normal volunteers: evidence for monoaminergic mechanisms. Neuropsychopharmacology 20:322-339.

Shepherd GM. 1996. The dendritic spine: a multifunctional integrative unit. J Neurophysiol 75:2197-2210.

Sklair-Tavron L, Shi W-X, Lane SB, Harris HW, Bunney BS, Nestler EJ. 1996. Chronic morphine induces visible changes in the morphology of mesolimbic dopamine neurons. Proc Natl Acad Sci USA 93:11202-11207.

Smith JE, Dworkin SI. 1990. Behavioral contingencies determine changes in drug-induced transmitter turnover. Drug Dev Res 20: 337-348

Stefanski R, Ladenheim B, Lee SH, Cadet JL, Goldberg SR. 1999. Neuroadaptations in the dopaminergic system after active selfadministration but not after passive administration of methamphetamine. Eur J Pharmacol 371:123-135.

Weeks JR. 1972. Long-term intravenous infusions. In: Meyers RD, editor. Methods in psychobiology. London: Academic Press. p 155168.

Wilson JM, Nobrega JN, Corrigall WA, Coen KM, Shannak K, Kish SJ. 1994. Amygdala dopamine levels are markedly elevated after self- but not passive-administration of cocaine. Brain Res 668:39 45 .

Woolley CS. 1999. Electrophysiological and cellular effects of estrogen on neuronal function. Crit Rev Neurobiol 13:1-20.

Zilles K. 1985. The cortex of the rat. Berlin: Springer-Verlag.

Zilles K, Wree A, Dausch N-D. 1990. Anatomy of the neocortex: neurochemical organization. In: Kolb B, Tees RC, editors. The cerebral cortex of the rat. Cambridge, MA: MIT Press. p 113-150. 